\title{
BMJ
}

\section{Enterovirus infection and type 1 diabetes mellitus: systematic review and meta-analysis of observational molecular studies}

\author{
Wing-Chi G Yeung, medical student,' William D Rawlinson, professor, 1,2 Maria E Craig, associate professor ${ }^{2,3,4}$
}

${ }^{1}$ Faculty of Medicine, University of New South Wales, Sydney, NSW 2052, Australia

${ }^{2}$ Virology Research, POWH and UNSW Research Laboratories, Prince of Wales Hospital, Randwick, NSW 2031

${ }^{3}$ The Children's Hospital at Westmead, Institute of Endocrinology and Diabetes, Sydney

${ }^{4}$ Discipline of Paediatrics and Child Health, University of Sydney, Sydney

Correspondence to: $\mathrm{M}$ Craig, Institute of Endocrinology and Diabetes, The Children's Hospital at Westmead, Locked Bag 4001, Westmead NSW 2145, Australia m.craig@unsw.edu.au

\section{ABSTRACT}

Objective To review the association between current enterovirus infection diagnosed with molecular testing and development of autoimmunity or type 1 diabetes. Design Systematic review and meta-analysis of observational studies, analysed with random effects models.

Data sources PubMed (until May 2010) and Embase (unti May 2010), no language restrictions, studies in humans only; reference lists of identified articles; and contact with authors.

Study eligibility criteria Cohort or case-control studies measuring enterovirus RNA or viral protein in blood, stool, or tissue of patients with pre-diabetes and diabetes, with adequate data to calculate an odds ratio and $95 \%$ confidence intervals.

Results The 24 papers and two abstracts (all case-control studies) that met the eligibility criteria included 4448 participants. Study design varied greatly, with a high level of statistical heterogeneity. The two separate outcomes were diabetes related autoimmunity or type 1 diabetes. Meta-analysis showed a significant association between enterovirus infection and type 1 diabetes related autoimmunity (odds ratio 3.7, 95\% confidence interval 2.1 to 6.8 ; heterogeneity $\left.\mathrm{X}^{2} / \mathrm{df}=1.3\right)$ and clinical type 1 diabetes $\left(9.8,5.5\right.$ to $\left.17.4 ; \mathrm{X}^{2} / \mathrm{df}=3.2\right)$.

Conclusions There is a clinically significant association between enterovirus infection, detected with molecular methods, and autoimmunity/type 1 diabetes. Larger prospective studies would be needed to establish a clear temporal relation between enterovirus infection and the development of autoimmunity and type 1 diabetes.

\section{INTRODUCTION}

Type 1 diabetes is believed to result from a complex interplay between genetic predisposition, the immune system, and environmental factors. ${ }^{1}$ In recent decades there has been a rapid rise in the incidence of childhood type 1 diabetes worldwide, especially in those under the age of 5..$^{2-6}$ In Europe, from 1989-2003 the average annual increase was $3.9 \%$, too fast to be accounted for by genetics alone. ${ }^{4}$ Evidence in support of a putative role for viral infections in the development of type 1 diabetes comes from epidemiological studies that have shown a significant geographical variation in incidence, a seasonal pattern to disease presentation, ${ }^{2378}$ and an increased incidence of diabetes after enterovirus epidemics. ${ }^{9}$

Enteroviruses are perhaps the most well studied environmental factor in relation to type 1 diabetes. A possible link was first reported by Gamble et al in $1969,{ }^{10}$ with many subsequent studies, in humans and animal models of diabetes, showing an association, particularly with coxsackievirus B-4. Higher rates of enterovirus infection, defined by detection of enterovirus IgM or IgG, or both, viral RNA with reverse transcription polymerase chain reaction (RT PCR), and viral capsid protein, have been found in patients with diabetes at diagnosis compared with controls. ${ }^{11-17}$ Prospective studies have also shown more enterovirus infections in children who developed islet autoantibodies or subsequent diabetes, or both; as well as a temporal relation between infection and autoimmunity. ${ }^{1318-20}$

The relation between enterovirus infection and diabetes is not consistent across all studies, ${ }^{21-24}$ however, and the subject remains controversial. ${ }^{25}$ Furthermore, in animal models viral infections might also protect from diabetes. ${ }^{25}$ A systematic review of coxsackie B virus serological studies did not show an association with type 1 diabetes, ${ }^{26}$ but to date there has been no systematic review of molecular studies. Based on the hypothesis that enterovirus infection increases the risk of pancreatic islet autoimmunity or type 1 diabetes, or both, we carried out a systematic review of controlled studies that used molecular virological methods to investigate the association between enteroviruses and type 1 diabetes.

\section{METHODS}

Two reviewers (WGY and MEC) independently conducted a systematic search for controlled observational studies of enterovirus and type 1 diabetes mellitus. Databases searched were PubMed (from 1965 to May 2010) and Embase (from 1974 to May 2010). Search terms (exploded, all subheadings) used were: 'diabetes mellitus', 'enterovirus', 'coxsackievirus', 'ECHOvirus', 'polymerase chain reaction', 'PCR', 'RNA', 
Table 1|Summary of molecular studies investigating pre-diabetes and enteroviruses

\begin{tabular}{|c|c|c|c|c|c|c|c|c|}
\hline Study & Country & $\begin{array}{l}\text { Cases/ } \\
\text { controls }\end{array}$ & Cases & $\begin{array}{c}\text { Autoantibodies } \\
\text { detected }\end{array}$ & Age in cases & Controls & $\begin{array}{l}\text { Method of } \\
\text { detection }\end{array}$ & EV type sequenced \\
\hline $\begin{array}{l}\text { Al-Shaheeb, } \\
2010^{30}\end{array}$ & Australia & $13 / 198$ & $\begin{array}{l}\text { Autoantibody positive } \\
\text { children with first } \\
\text { degree relative with } \\
\text { T1DM }\end{array}$ & $\begin{array}{l}\text { At least two of ICA, } \\
\text { GADA, IA2A, or IAA }\end{array}$ & $\begin{array}{l}\text { Birth cohort from VIGR } \\
\text { study }\end{array}$ & $\begin{array}{l}\text { Children from same } \\
\text { cohort negative for } \\
\text { autoantibody }\end{array}$ & $\begin{array}{l}\text { EV RNA in serum } \\
\text { (RT-PCR) }\end{array}$ & - \\
\hline Coutant, $2002^{32}$ & France & $5 / 49$ & $\begin{array}{l}\text { Autoantibody positive } \\
\text { siblings of probands } \\
\text { with diabetes }\end{array}$ & ICA, GADA & Age $2.4-16.5$ & $\begin{array}{l}\text { Healthy children } \\
\text { matched for age, sex, } \\
\text { place, and sampling } \\
\text { date }\end{array}$ & $\begin{array}{l}\text { EV RNA in serum } \\
\text { (RT-PCR) }\end{array}$ & - \\
\hline Graves, $2003^{22}$ & USA & $13 / 26$ & $\begin{array}{l}\text { Autoantibody positive } \\
\text { (eventual); sibling } \\
\text { offspring cohort }\end{array}$ & $\begin{array}{l}\text { At least one of IAA, } \\
\text { GADA, or ICA }\end{array}$ & $\begin{array}{l}\text { From DAISY cohort } \\
\text { study, children at } \\
\text { moderate to high risk of } \\
\text { developing T1DM }\end{array}$ & $\begin{array}{l}\text { Age matched children } \\
\text { from same cohort } \\
\text { negative for } \\
\text { autoantibody }\end{array}$ & $\begin{array}{l}\text { EV RNA in serum, } \\
\text { saliva, and rectal } \\
\text { swab (RT-PCR) }\end{array}$ & - \\
\hline $\begin{array}{l}\text { Moya-Suri, } \\
2005^{33}\end{array}$ & Germany & $50 / 50$ & Autoantibody positive & $\begin{array}{l}\text { At least one of IAA, } \\
\text { GADA, ICA, or IA2A }\end{array}$ & $\begin{array}{l}\text { Median age 12, } \\
\text { IQR 10-14 }\end{array}$ & $\begin{array}{l}\text { Children from same } \\
\text { cohort negative for } \\
\text { autoantibody }\end{array}$ & $\begin{array}{l}\text { EV RNA in serum } \\
\text { (RT-PCR) }\end{array}$ & $\begin{array}{l}\text { CVB-4, CVB-2, } \\
\text { CVB-6 }\end{array}$ \\
\hline $\begin{array}{l}\text { Salminen, } \\
2003^{20}\end{array}$ & Finland & $41 / 196$ & $\begin{array}{l}\text { Autoantibody positive } \\
\text { children (samples taken } \\
6 \text { months before } \\
\text { seroconversion) }\end{array}$ & $\begin{array}{l}\text { At least one of ICA, } \\
\text { GADA, IAA, or IA2A }\end{array}$ & $\begin{array}{l}\text { Birth cohort from DIPP } \\
\text { study }\end{array}$ & $\begin{array}{l}\text { Children from same } \\
\text { cohort negative for } \\
\text { autoantibody }\end{array}$ & $\begin{array}{l}\text { EV RNA in serum } \\
\text { (RT-PCR) }\end{array}$ & - \\
\hline $\begin{array}{l}\text { Sadeharju, } \\
2003^{28}\end{array}$ & Finland & $19 / 84$ & $\begin{array}{l}\text { Autoantibody positive } \\
\text { (eventual), from Trial to } \\
\text { Reduce IDDM in } \\
\text { Genetically at Risk } \\
\text { (TRIGR) study }\end{array}$ & $\begin{array}{l}\text { At least one of IAA, } \\
\text { GADA, or IA2A }\end{array}$ & $\begin{array}{l}\text { Birth cohort from TRIGR } \\
\text { study }\end{array}$ & $\begin{array}{l}\text { Children from same } \\
\text { study cohort negative } \\
\text { for autoantibody and } \\
\text { matched for sex, HLA, } \\
\text { and intervention group }\end{array}$ & $\begin{array}{l}\text { EV RNA in serum } \\
\text { (RT-PCR) }\end{array}$ & - \\
\hline $\begin{array}{l}\text { Salminen, } \\
2004^{29}\end{array}$ & Finland & $12 / 53$ & $\begin{array}{l}\text { Autoantibody positive } \\
\text { (eventual) }\end{array}$ & At least one & $\begin{array}{l}\text { Birth cohort from DIPP } \\
\text { study }\end{array}$ & $\begin{array}{l}\text { Children from same } \\
\text { study cohort negative } \\
\text { for autoantibody } \\
\text { (matched for age, sex, } \\
\text { and HLA DQ haplotype) }\end{array}$ & $\begin{array}{l}\text { EV RNA in stool } \\
\text { samples } \\
\text { (RT-PCR) and/or } \\
\text { serum }\end{array}$ & $\begin{array}{l}\text { PV-3, CVA-9, CVB-3, } \\
\text { CVB-4, CVB-5, EV-3, } \\
\text { EV-11, EV-18, } \\
\text { EV-24, EV-25 }\end{array}$ \\
\hline $\begin{array}{l}\text { Sarmiento, } \\
2007^{16}\end{array}$ & Cuba & $32 / 63$ & $\begin{array}{l}\text { First degree relatives } \\
\text { with ICA positive T1DM }\end{array}$ & ICA & $\begin{array}{l}\text { Mean age 13.5 (SD 9.5), } \\
\text { range 1-46 }\end{array}$ & $\begin{array}{l}\text { Healthy people verified } \\
\text { negative for ICA with no } \\
\text { family history of } \\
\text { diabetes }\end{array}$ & $\begin{array}{l}\text { EV RNA in serum } \\
\text { (RT-PCR) }\end{array}$ & - \\
\hline
\end{tabular}

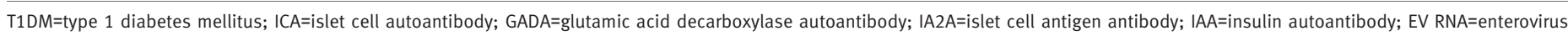
RNA; RT PCA=reverse transcription-polymerase chain reaction; IQR=interquartile range.

'DNA', 'nucleic acid', and 'capsid protein'. The search was limited to studies in humans in any language and was supplemented by hand searching reference lists in the identified papers and by direct contact with authors.

Studies were eligible for inclusion if they were casecontrol or cohort studies (including those published as letters or abstracts); measured enterovirus RNA or viral capsid protein in blood, stool, or tissue of patients with pre-diabetes and diabetes; and provided adequate data to enable calculation of odds ratios and 95\% confidence intervals. No restrictions were placed on the study population. We included only those studies that used molecular methods for viral detection (such as RT-PCR (reverse transcription-polymerase chain reaction), in situ hybridisation, or immunostaining for detection of viral capsid protein) to identify current or recent infection and because molecular testing is now standard for diagnosis of acute enterovirus infection.

The results of identified studies were classified into two groups, pre-diabetes and diabetes, depending on whether autoimmunity or type 1 diabetes was the outcome. There were four main categories of cases: autoantibody positive, newly diagnosed type 1 diabetes, established type 1 diabetes, and eventual type 1 diabetes. The latter three were combined into the diabetes group.

We calculated unadjusted odds ratios with 95\% confidence intervals and $\mathrm{P}$ values for enterovirus identification in patients with pre-diabetes versus no diabetes and patients with diabetes versus no diabetes from the published figures using the Mantel-Haenszel method. The analysis was performed with both fixed and random effects models. Because of the presence of significant heterogeneity we have presented only the results from random effects models. Combined odds ratios were also calculated for different subgroups of studies according to study design. Statistical heterogeneity was explored with Cochrane's Q test and the $\mathrm{I}^{2}$ statistic, which provides the relative amount of variance of the summary effect caused by heterogeneity between studies.

We assessed study quality using the NewcastleOttawa quality assessment scale (NOS) for casecontrol studies, as recommended by Cochrane collaboration. ${ }^{27}$ Three areas were evaluated-selection, comparability, and exposure - giving a possible total score 9 , with 5 or more classed as good methods. In the comparability category, studies were assessed as to whether they controlled for age and sampling time 
Table 2 |Summary of molecular studies investigating type 1 diabetes (T1DM) and enteroviruses

\begin{tabular}{|c|c|c|c|c|c|c|c|}
\hline Study & Country & $\begin{array}{l}\text { Cases/ } \\
\text { controls }\end{array}$ & Cases and details of diabetes & $\begin{array}{c}\text { Age of cases (years unless } \\
\text { specified) }\end{array}$ & Controls & Method of detection & $\begin{array}{l}\text { EV type } \\
\text { sequenced }\end{array}$ \\
\hline \multirow{2}{*}{$\begin{array}{l}\text { Andreolet- } \\
\text { ti, } 1997^{14}\end{array}$} & \multirow[b]{2}{*}{ France } & \multirow[b]{2}{*}{$12 / 15$} & $\begin{array}{l}\text { Newly diagnosed with metabolic } \\
\text { decompensation }\end{array}$ & Mean 28.2 (SD 10.4) & \multirow[b]{2}{*}{ Healthy adults } & \multirow{2}{*}{$\begin{array}{l}\text { EV RNA in peripheral blood } \\
\text { (RT PCR) }\end{array}$} & \multirow[b]{2}{*}{$\begin{array}{l}\text { CVB-3, CVB- } \\
4\end{array}$} \\
\hline & & & $\begin{array}{l}\text { Previously diagnosed with } \\
\text { metabolic decompensation }\end{array}$ & Mean 32.6 (SD 13.3) & & & \\
\hline $\begin{array}{l}\text { Buesa- } \\
\text { Gomez, } \\
1994^{60}\end{array}$ & USA & $2 / 5$ & Fatal acute onset & 14 months and 3 years & $\begin{array}{l}\text { Children who died from } \\
\text { non-diabetic causes }\end{array}$ & $\begin{array}{l}\text { Coxsackie RNA in autopsy } \\
\text { pancreatic samples (RT PCR) }\end{array}$ & - \\
\hline $\begin{array}{l}\text { Clements, } \\
1995^{41}\end{array}$ & UK & $14 / 45$ & Newly diagnosed & Mean 3.9, range $1.4-6.0$ & $\begin{array}{l}\text { Normal subjects matched for age, } \\
\text { sex, sample date, and place }\end{array}$ & EV RNA in serum (RT PCR) & $\begin{array}{l}\text { CVB-3, CVB- } \\
4\end{array}$ \\
\hline $\begin{array}{l}\text { Coutant, } \\
2002^{32}\end{array}$ & France & $16 / 49$ & $\begin{array}{l}\text { Newly diagnosed (within } 1 \text { month } \\
\text { of diagnosis) }\end{array}$ & Range $<6$ & $\begin{array}{l}\text { Healthy children matched for age, } \\
\text { sex, sample date, and place }\end{array}$ & EV RNA in serum (RT PCR) & - \\
\hline $\begin{array}{l}\text { Craig, } \\
2003^{58}\end{array}$ & Australia & $206 / 160$ & $\begin{array}{l}\text { Newly diagnosed (within } 2 \text { weeks } \\
\text { of diagnosis) }\end{array}$ & Median 8.2, range $0.7-15.7$ & $\begin{array}{l}\text { Children without diabetes from } \\
\text { community }\end{array}$ & $\begin{array}{l}\text { EV RNA in plasma or stool } \\
\text { samples (RT PCR) }\end{array}$ & EV-71 \\
\hline $\begin{array}{l}\text { Dahlquist, } \\
2004^{36}\end{array}$ & Sweden & $600 / 600$ & $\begin{array}{l}\text { Eventual diabetes, on Swedish } \\
\text { childhood diabetes register }\end{array}$ & Neonate & $\begin{array}{l}\text { People without diabetes from } \\
\text { same biobank }\end{array}$ & $\begin{array}{l}\text { EV RNA in newborn blood spots } \\
\text { (RT PCR) }\end{array}$ & - \\
\hline $\begin{array}{l}\text { Dotta, } \\
2007^{46}\end{array}$ & Italy & $6 / 26$ & Recent onset & Range $14-50$ & Normal multi-organ donors & $\begin{array}{l}\text { EV vp1 immunostaining in } \\
\text { autopsy pancreatic samples } \\
\text { (Dako anti-vp1) }\end{array}$ & CVB-4 \\
\hline $\begin{array}{l}\text { Foulis, } \\
1990^{38}\end{array}$ & UK & $147 / 43$ & $\begin{array}{l}88 \text { recent onset (duration }<1 \text { year } \\
\text { ), } 59 \text { established (duration } \\
1-19 \text { years) }\end{array}$ & Range 1-37 & $\begin{array}{l}\text { Normal autopsy pancreases from } \\
11 \text { neonates, } 21 \text { children, } 11 \\
\text { adults }\end{array}$ & $\begin{array}{l}\text { EV vp1 immunostaining in } \\
\text { autopsy pancreatic samples }\end{array}$ & - \\
\hline \multirow{2}{*}{$\begin{array}{l}\text { Foy, } \\
1995^{35}\end{array}$} & \multirow[t]{2}{*}{ UK } & $17 / 42$ & $\begin{array}{l}\text { Newly diagnosed (on day of } \\
\text { diagnosis) }\end{array}$ & Median 11 , range $2-35$ & \multirow{2}{*}{$\begin{array}{l}\text { Patients without diabetes, } \\
\text { matched for age and sex }\end{array}$} & \multirow{2}{*}{$\begin{array}{l}\text { EV RNA in peripheral blood } \\
\text { (RT PCR) }\end{array}$} & \multirow[t]{2}{*}{-} \\
\hline & & $38 / 42$ & Duration 2 months-10 years & Median 11 , range 3-16 & & & \\
\hline $\begin{array}{l}\text { Kawashi- } \\
\text { ma } 2004^{61}\end{array}$ & Japan & $61 / 58$ & Type 1 diabetes & Range 9 months - 40 years & Healthy people & EV RNA in serum (RT PCR) & $\begin{array}{l}\text { CVB-2, CVB- } \\
3, \text { CVB-4, } \\
\text { CVB-5 }\end{array}$ \\
\hline \multirow{2}{*}{$\begin{array}{l}\text { Lönnrot, } \\
2000^{31}\end{array}$} & \multirow{2}{*}{ Finland } & $11 / 34$ & $\begin{array}{l}\text { Eventual diabetes, from DiMe } \\
\text { Study }\end{array}$ & Mean 8.4 , range $2.6-17$ & \multirow{2}{*}{$\begin{array}{l}\text { Children from same study cohort } \\
\text { who did not develop T1DM or } \\
\text { autoantibodies }\end{array}$} & \multirow{2}{*}{ EV RNA in serum (RT PCR) } & \multirow[t]{2}{*}{-} \\
\hline & & $47 / 34$ & Newly diagnosed & Mean 4.4 & & & \\
\hline \multirow{2}{*}{$\begin{array}{l}\text { Maha, } \\
2003^{34}\end{array}$} & \multirow{2}{*}{ Egypt } & $40 / 30$ & Recent onset («1 year) & Mean 11.30 (SD 2.16) & \multirow{2}{*}{-Normal healthy children } & \multirow{2}{*}{$\begin{array}{l}\text { EV RNA in serum (RT PCR via } \\
\text { tissue culture) }\end{array}$} & \multirow{2}{*}{$\begin{array}{l}\text { CVB-4, CVB- } \\
6\end{array}$} \\
\hline & & $30 / 30$ & Duration >1 year & Mean 11.80 (SD 2.70) & & & \\
\hline $\begin{array}{l}\text { Moya-Suri, } \\
2005^{33}\end{array}$ & Germany & $47 / 50$ & $\begin{array}{l}\text { Newly diagnosed (median } 5 \text { days } \\
\text { from diagnosis) }\end{array}$ & Median 13, IQR 11-15 & $\begin{array}{l}\text { Children from same study } \\
\text { negative autoantibodies }\end{array}$ & EV RNA in serum (RT PCR) & $\begin{array}{l}\text { CVB-4, CVB- } \\
2, \text { CVB-6 }\end{array}$ \\
\hline $\begin{array}{l}\text { Nairn, } \\
1999^{12}\end{array}$ & UK & $110 / 182$ & $\begin{array}{l}\text { Newly diagnosed (within } 1 \text { week } \\
\text { from diagnosis) }\end{array}$ & $\begin{array}{l}\text { Mean } 7.1 \text {, range } 3 \text { months } \\
-16 \text { years }\end{array}$ & $\begin{array}{l}\text { Children without diabetes } \\
\text { (matched for age, location, time } \\
\text { of sampling) }\end{array}$ & EV RNA in serum (RT PCR) & $\begin{array}{l}\text { PV1-3, CVA- } \\
21, \text { CVA-24, } \\
\text { EV-70 }\end{array}$ \\
\hline $\begin{array}{l}\text { Oikarinen, } \\
2007^{39}\end{array}$ & Finland & $12 / 10$ & $\begin{array}{l}\text { Established (duration 0-51 years, } \\
\text { median 13) }\end{array}$ & Median 30, range 18-53 & $\begin{array}{l}\text { Patients without diabetes from } \\
\text { same hospital department }\end{array}$ & $\begin{array}{l}\text { vp1 immunostaining in small } \\
\text { bowel mucosa (Dako anti-vp1) }\end{array}$ & - \\
\hline $\begin{array}{l}\text { Richard- } \\
\text { son, } \\
2009^{17}\end{array}$ & UK & $72 / 119$ & $\begin{array}{l}\text { Recent onset (8.2 (SD 4.1) } \\
\text { months from diagnosis) }\end{array}$ & $\begin{array}{l}\text { Mean } 12.65(\mathrm{SD} 1.1) \\
\text { range } 1-42\end{array}$ & $\begin{array}{l}\text { Normal autopsy pancreases from } \\
11 \text { neonates, } 39 \text { children and } 69 \\
\text { adults }\end{array}$ & $\begin{array}{l}\text { EV vp1 immunostaining in } \\
\text { autopsy pancreatic samples } \\
\text { (Dako anti-vp1) }\end{array}$ & - \\
\hline $\begin{array}{l}\text { Sarmiento, } \\
2007^{16}\end{array}$ & Cuba & $34 / 68$ & $\begin{array}{l}\text { Newly diagnosed (0.78 (SD 2.4) } \\
\text { days from diagnosis) }\end{array}$ & $\begin{array}{l}\text { Mean } 7.3(\mathrm{SD} 4.5) \text {, } \\
\text { range 1-15 }\end{array}$ & $\begin{array}{l}\text { Healthy subjects, verified ICA } \\
\text { negative and no family history of } \\
\text { diabetes }\end{array}$ & EV RNA in serum (RT PCR) & - \\
\hline $\begin{array}{l}\text { Schulte, } \\
2010^{43}\end{array}$ & $\begin{array}{l}\text { Nether- } \\
\text { lands }\end{array}$ & $10 / 20$ & $\begin{array}{l}\text { Newly diagnosed (within } 1 \text { month } \\
\text { of diagnosis) }\end{array}$ & Mean 9.7 , range $5-14$ & $\begin{array}{l}\text { Children of same age range in } \\
\text { hospital with non-endocrine } \\
\text { disorders }\end{array}$ & $\begin{array}{l}\text { EV RNA in peripheral blood } \\
\text { mononuclear cells (RT PCR) }\end{array}$ & HEV-B \\
\hline $\begin{array}{l}\text { Toniolo, } \\
2010^{44}\end{array}$ & Italy & $112 / 58$ & Newly diagnosed & $\begin{array}{l}\text { Mean } 6.8 \text {, median 9.0, } \\
\text { range } 2-16\end{array}$ & Healthy children & $\begin{array}{l}\text { EV RNA in peripheral blood } \\
\text { (RT PCR) }\end{array}$ & $\begin{array}{l}\text { HEV-A, HEV- } \\
\text { B, HEV-C, } \\
\text { HEV-D }\end{array}$ \\
\hline $\begin{array}{l}\text { Yin, } \\
2002^{40}\end{array}$ & Sweden & $24 / 24$ & $\begin{array}{l}\text { Newly diagnosed (within } 1 \text { week } \\
\text { from diagnosis) }\end{array}$ & Mean 8.4 , range $1.6-15.7$ & $\begin{array}{l}\text { Healthy children from nearby } \\
\text { counties }\end{array}$ & EV RNA in PBMCs (RT PCR) & $\begin{array}{l}\text { CVB-5, EV-5, } \\
\text { CVB-4 }\end{array}$ \\
\hline $\begin{array}{l}\text { Ylipaasto, } \\
2004^{42}\end{array}$ & $\begin{array}{l}\text { Finland/ } \\
\text { Germany }\end{array}$ & $65 / 40$ & Duration: few weeks to 19 years & Range 18-52 & $\begin{array}{l}\text { Non-diabetic pancreases (age- } \\
\text { sex matched) }\end{array}$ & $\begin{array}{l}\text { EV RNA in autopsy pancreatic } \\
\text { samples (RNA probes and in situ } \\
\text { hybridisation) }\end{array}$ & - \\
\hline
\end{tabular}

EV RNA=enterovirus RNA; RT PCA=reverse transcription-polymerase chain reaction.

as these are the two factors most likely to affect the incidence of enterovirus infection.

\section{RESULTS}

Our search returned a total of 114 publications and abstracts. After review of titles and abstracts, we identified and included 25 relevant papers - two letters and 23 articles. We also included data from two studies published as abstracts only. All were case-control studies (six were nested case-control studies that used samples collected prospectively $\left.{ }^{202228-30}\right)$. One was excluded because it was a pilot study ${ }^{13}$ analysing the 
Table $3 \mid$ Quality of evidence in molecular studies investigating type 1 diabetes (T1DM) and enteroviruses

\begin{tabular}{|c|c|c|c|c|c|c|c|c|c|}
\hline \multirow[b]{2}{*}{ Study } & \multirow{2}{*}{$\begin{array}{l}\text { NHMRC level } \\
\text { of evidence* }\end{array}$} & \multirow{2}{*}{$\begin{array}{l}\text { Newcastle- } \\
\text { Ottawa scale } \\
\text { score }\end{array}$} & \multirow{2}{*}{$\begin{array}{l}\text { Diagnostic criteria for } \\
\text { autoimmunity and/or } \\
\text { type } 1 \text { diabetes given? }\end{array}$} & \multicolumn{5}{|c|}{ Cases and controls matched? } & \multirow{2}{*}{$\begin{array}{l}\text { Details of vira } \\
\text { detection } \\
\text { given? }\end{array}$} \\
\hline & & & & Age & Sex & HLA & Place & Sample time & \\
\hline $\begin{array}{l}\text { Andreoletti, } \\
1997^{14}\end{array}$ & III-3 & 4 & No & No & No & No & No & No & $\begin{array}{c}\text { Yes } \\
\text { (referenced) }\end{array}$ \\
\hline $\begin{array}{l}\text { Al-Shaheeb, } \\
2010^{30}\end{array}$ & II & 7 & Yes & No & No & No & Yes & Yes & Yes \\
\hline $\begin{array}{l}\text { Buesa-Gomez, } \\
1994^{60}\end{array}$ & III-3 & 4 & No & No & No & No & No & No & Yes \\
\hline $\begin{array}{l}\text { Clements, } \\
1995^{41}\end{array}$ & III-3 & 6 & No & Yes & Yes & No & Yes & Yes & No \\
\hline Coutant, $2002^{32}$ & III-3 & 6 & No & Yes & Yes & No & Yes & Yes & Yes \\
\hline Craig, $2003^{58}$ & III-3 & 6 & Yes (diabetes register) & No & No & No & No & Yes & Yes \\
\hline $\begin{array}{l}\text { Dahlquist, } \\
2004^{36}\end{array}$ & II & 7 & Yes (diabetes register) & Yes & No & No & No & No & $\begin{array}{c}\text { Yes } \\
\text { (referenced) }\end{array}$ \\
\hline Dotta, $2007^{46}$ & III-3 & 5 & No & No & No & No & No & NA & Yes \\
\hline Foulis, $1990^{38}$ & $111-3$ & 3 & No & No & No & No & No & NA & Yes \\
\hline Foy, $1995^{35}$ & III-3 & 6 & Yes & Yes & Yes & No & No & No & Yes \\
\hline Graves, $2003^{22}$ & II & 7 & $\begin{array}{l}\text { Yes for autoimmunity, } \\
\text { no for diabetes }\end{array}$ & Yes & No & No & No & No & No \\
\hline $\begin{array}{l}\text { Kawashima, } \\
2004^{61}\end{array}$ & III-3 & 5 & No & No & No & No & No & No & Yes \\
\hline Lönnrot, $2000^{31}$ & II & 6 & No & Yes & Yes & Yes & No & Yes & Yes \\
\hline Maha, $2003^{34}$ & III-3 & 5 & No & No & No & No & No & No & Yes \\
\hline $\begin{array}{l}\text { Moya-Suri, } \\
2005^{33}\end{array}$ & III-3 & 7 & $\begin{array}{l}\text { Yes for autoimmunity, } \\
\text { no for diabetes }\end{array}$ & Yes & Yes & No & No & No & Yes \\
\hline Nairn, $1999^{12}$ & III-3 & 7 & No & Yes & No & No & Yes & Yes & $\begin{array}{c}\text { Yes } \\
\text { (referenced) }\end{array}$ \\
\hline $\begin{array}{l}\text { Oikarinen, } \\
2007^{39}\end{array}$ & III-3 & 4 & No & No & No & No & No & NA & Yes \\
\hline $\begin{array}{l}\text { Richardson, } \\
2009^{17}\end{array}$ & $|I|-3$ & 4 & No & No & No & No & No & NA & Yes \\
\hline $\begin{array}{l}\text { Sadeharju, } \\
2003^{28}\end{array}$ & II & 8 & Yes & Yes & Yes & Yes & No & Yes & $\begin{array}{c}\text { Yes } \\
\text { (referenced) }\end{array}$ \\
\hline $\begin{array}{l}\text { Salminen, } \\
2003^{20}\end{array}$ & II & 6 & Yes & Yes & Yes & Yes & No & No & $\begin{array}{c}\text { Yes } \\
\text { (referenced) }\end{array}$ \\
\hline $\begin{array}{l}\text { Salminen, } \\
2004^{29}\end{array}$ & II & 7 & Yes & Yes & Yes & Yes & No & Yes & Yes \\
\hline $\begin{array}{l}\text { Sarmiento, } \\
2007^{16}\end{array}$ & || $\mid-3$ & 6 & No & Yes & Yes & No & Yes & Yes & Yes \\
\hline Schulte, $2010^{43}$ & III-3 & 4 & No & No & No & No & No & No & Yes \\
\hline Toniolo, $2010^{44}$ & III-3 & 7 & Yes & No & No & No & Yes & No & Yes \\
\hline Yin, $2002^{40}$ & $111-3$ & 7 & No & Yes & Yes & No & Yes & No & Yes \\
\hline $\begin{array}{l}\text { Ylipaasto, } \\
2004^{42}\end{array}$ & III-3 & 5 & No & Yes & Yes & No & No & No & Yes \\
\hline
\end{tabular}

same data as a duplicate publication. ${ }^{20}$ Of the 26 remaining studies, eight contained more than one case group $^{14162231-35}$ and these were analysed separately, giving a total of 34 studies. Of these, nine were studies of pre-diabetes (198 cases and 733 controls) and 25 were studies of diabetes (1733 cases and 1784 controls).

\section{Characteristics of included studies}

Thirty studies used RT-PCR or in situ hybridisation to detect enterovirus RNA, while four performed immunostaining for the enterovirus capsid protein vp1 on autopsy pancreas specimens (tables 1 and 2). Within the pre-diabetes group, all except two of the studies defined autoimmunity as positivity for at least one autoantibody associated with type 1 diabetes (table 1). Study populations varied in age distribution. While most studies investigated children and adolescents (aged 16 and below), some included adults up to age 53

\section{Quality of evidence}

The Newcastle-Ottawa scores ranged from 3 to 8, with 24 studies scoring 5 or more (table 3), indicating reasonably good methodological quality overall, with no studies reporting a non-response rate.

\section{Pre-diabetes}

Figure 1 presents the individual and summary odds ratio of the nine pre-diabetes studies. Odds ratios 


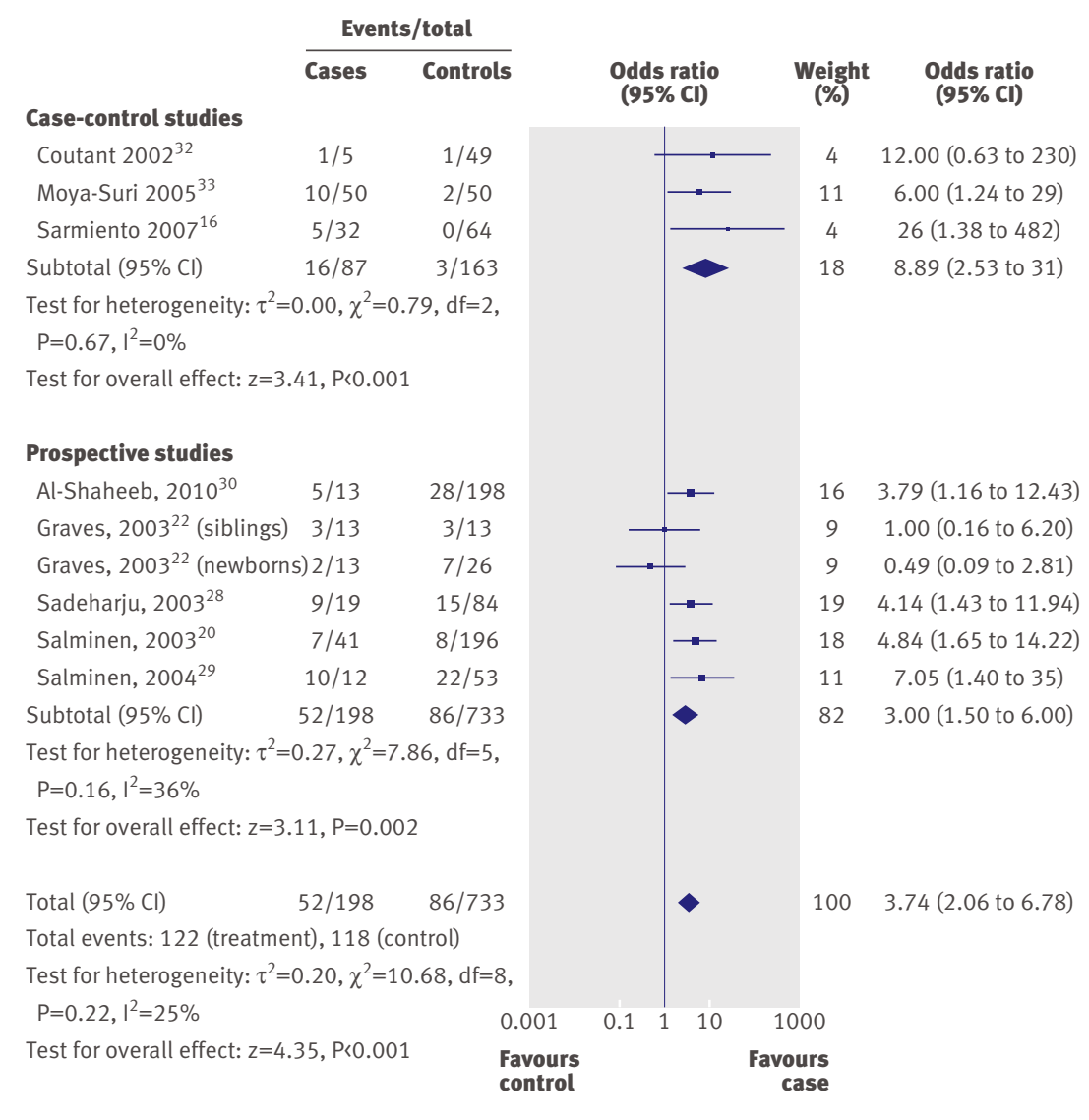

Fig 1| Odds ratios for enterovirus positivity in patients with pre-diabetes versus no diabetes

ranged from 0.1 to 483 , with a summary odds ratio of 3.7 (95\% confidence interval 2.1 to $6.8 ; \mathrm{P}<0.001$ ). There was some evidence for heterogeneity across the studies $\left(\chi^{2} / \mathrm{df}=1.34\right)$, but this value did not reach significance $(\mathrm{P}=0.22)$. When we analysed the results from the six nested case-control studies separately, the summary odds ratio was $3.0(1.5$ to $6.0 ; \mathrm{P}=0.002)$ (table 4).

Three of the nested case-control studies also separately examined the six or 12 month period preceding the first appearance of autoantibodies. ${ }^{2022}$ The summary odds ratio was 3.6 (1.3 to $9.8 ; \mathrm{P}=0.01)$. Five studies also sequenced the HLA haplotypes of their participants and two included those with low risk HLA genotypes. For those with high HLA risk haplotypes (five studies, 112 cases, 551 controls), the combined odds ratio was 3.5 (1.7 to $7.1 ; \mathrm{P}<0.001) .{ }^{20228-30}$ Only two studies (21 cases, 158 controls) included participants with low risk HLA genotypes, with conflicting results $(0.4,(0.04$ to $4.8)^{22}$ and $\left.9.3(1.9 \text { to } 45)^{30}\right)$, but the combined odds ratio was not significant $(2.3,0.1$ to $56 ; \mathrm{P}=0.62)$.

Table 4 |Combined odds ratios for pre-diabetes studies stratified by study type

\begin{tabular}{lcccc} 
Type of study & No of studies & Combined OR $(95 \% \mathrm{Cl})$ & $\mathrm{P}$ value & $\mathrm{X}^{2} / \mathrm{df*}$ \\
All & 9 & $3.7(2.1$ to 6.8$)$ & $<0.001$ & 1.34 \\
\hline Nested case-control studies & 6 & $3.0(1.5$ to 6.0$)$ & 0.002 & 1.57 \\
\hline Studies in Europe & 5 & $5.2(2.8$ to 9.6$)$ & $<0.001$ & 0.17
\end{tabular}

${ }^{*}$ Cochrane $x^{2}$ divided by degrees of freedom. Values $>1$ indicate heterogeneity.
Type 1 diabetes

Figure 2 shows the individual and summary odds ratios of the 25 studies of patients with type 1 diabetes. All studies except one ${ }^{32}$ showed an odds ratio over 1 for enterovirus positivity in patients with diabetes. Odds ratios ranged from 0.24 to 129 , with a summary odds ratio of 10 ( 5.5 to $17 ; \mathrm{P}<0.001)$. There was significant heterogeneity across the studies $\left(\chi^{2} / \mathrm{df}=3.21 ; \mathrm{P}<0.001\right)$.

We carried out a subgroup analysis with respect to method of enterovirus detection (RNA or capsid protein) and case selection (newly diagnosed $v$ established $v$ eventual diabetes; table 5 , fig 2 ). The combined odds ratios for newly diagnosed, established, and eventual diabetes were 13 (6 to 25), 11 (4 to 29), and 1.25 (0.2 to $7)$, respectively. The combined odds ratio of studies that used RNA detection was 8.8 (4.7 to $17 ; \mathrm{P}<0.001$ ), while for studies that performed immunostaining for enterovirus capsid protein, the odds ratio was 15 (7.5 to 31 ). There was no significant heterogeneity across studies that measured enteroviral vp1 protein, probably because of the similarity in study design.

We used sensitivity analyses to test the robustness of the results by country and study quality. For the 19 studies conducted in Europe, ${ }^{12141731-3335-44}$ the combined odds ratio was 8.6 (4.3 to $17 ; \mathrm{P}<0.001$ ), with significant heterogeneity $\left(\chi^{2} / \mathrm{df}=3.75, \mathrm{P}<0.001\right)$. The odds ratio was comparatively higher for the non-European studies $(13.5,7.1$ to 26$)$, with low heterogeneity, though there was considerable overlap of the confidence intervals between the two groups. When we excluded the studies with poor methodological quality (Newcastle-Ottawa score $<5)$, the combined odds ratio was similar (8.9, 4.6 to $17 ; \mathrm{P}<0.001)$. Subgroup analysis by HLA genotype was not performed because none of the studies performed HLA genotyping on all cases and controls.

\section{DISCUSSION}

This systematic review of 33 prevalence studies, involving 1931 cases and 2517 controls, shows a clinically significant association between enterovirus infection and islet autoimmunity or type 1 diabetes. The association between enterovirus infection, detected with molecular methods, and diabetes was strong, with almost 10 times the odds of enterovirus infection in children at diagnosis of type 1 diabetes compared with controls $(9.8,5.5$ to 17.4$)$, while the odds of infection was also higher in children with pre-diabetes than in controls (3.7, 2.1 to 6.8$)$. There was some evidence for geographical differences; in non-European studies the odds ratio was 13.5 (7.1 to 25.8) compared with 8.6 (4.3 to 17.3) in European studies, though there was considerably overlap in the confidence intervals. While the findings from this meta-analysis of observational studies cannot prove that enterovirus infection has a causal role in pathogenesis of diabetes, the results provide additional support to the direct evidence of enterovirus infection in pancreatic tissue of individuals with type 1 diabetes. ${ }^{4546}$

\section{Strengths and weaknesses}

We made every effort to reduce potential bias in this review, through use of pre-defined inclusion criteria, 


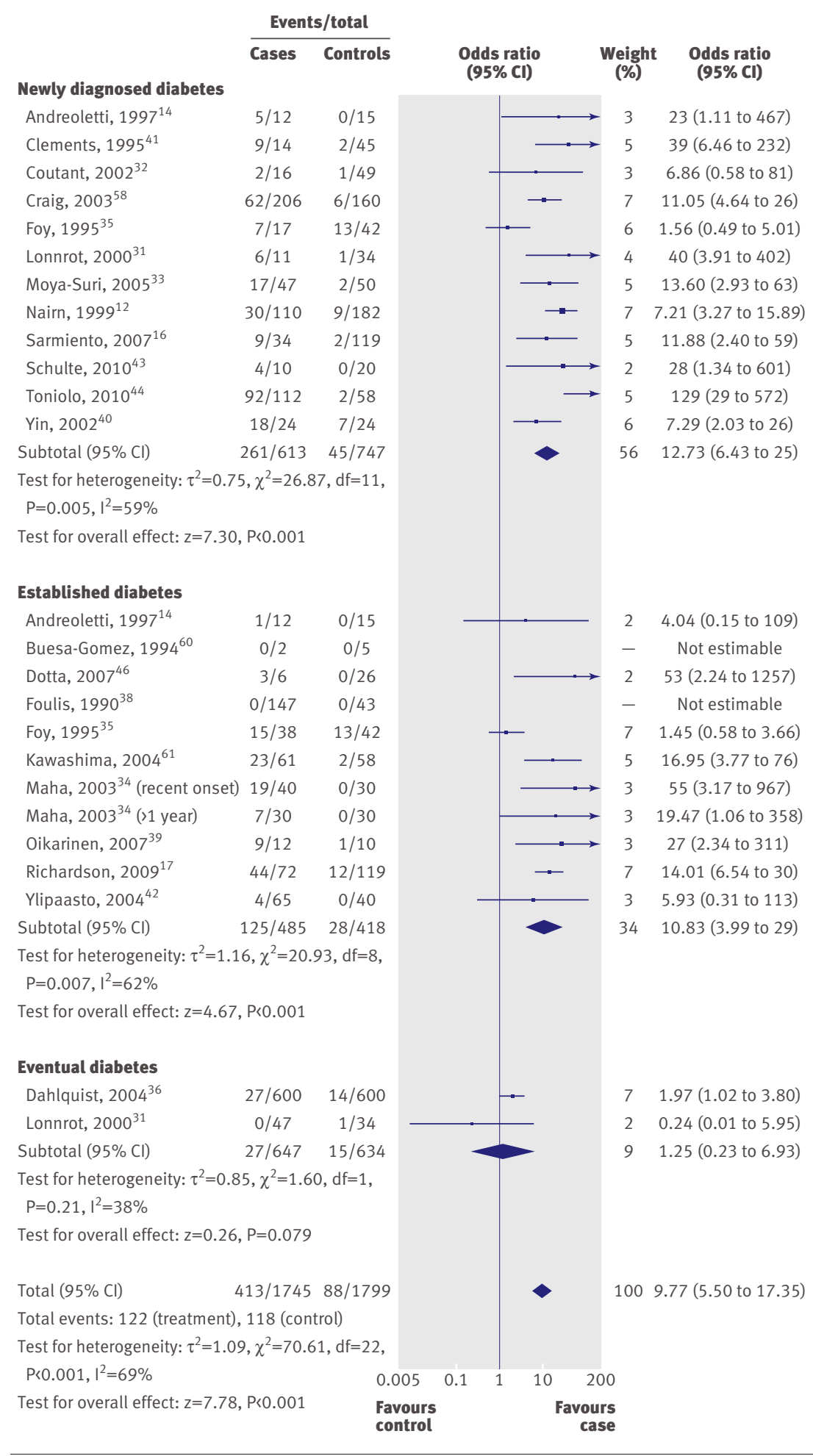

Fig 2 | Odds ratios for enterovirus positivity in patients with and without diabetes

independent searches by two reviewers, no language restriction, and searching of references lists and conference proceedings. We included studies in children and adults, reducing the risk of bias resulting from high rates background infection in children. ${ }^{478}$ Studies from throughout the world were included, reducing the risk of geographical bias related to infection rates. Most studies, however, were from European countries, where the incidence of type 1 diabetes is higher. Given the heterogeneity of the study populations, we used random effects models, providing more conservative effect estimates.

Several limitations could have influenced our findings, including factors inherent in a meta-analysis of observational studies. There was significant heterogeneity in study design and methods used. Only $10 \mathrm{stu}-$ dies matched for three or more potential confounding factors (age, genetic risk, geographical location, and sampling time). Most of the included studies used children without diabetes or who were negative for antibodies as controls, but there could have been unmeasured factors influencing their risk of developing diabetes. Other environmental factors might modify the risk of type 1 diabetes, such as cows' milk, ${ }^{49}$ vitamin $\mathrm{D},{ }^{50}$ and weight gain in infancy, ${ }^{51}$ but it is not possible to control for all of these potential confounders in case-control studies. Finally, enterovirus PCR primers had varying sensitivity and specificity, and not all studies reported the validation and limits of detection of their PCR method. Samples were obtained from various sites (serum, stool, throat swabs, etc) and because enteroviruses invade and replicate at mucosal surfaces, detection rates are likely to be higher in samples obtained from the gastrointestinal tract. ${ }^{5253}$

The overall methodological quality of the studies of the studies was relatively good, with 26 publications scoring 5 or more of 9 on the Newcastle-Ottawa scale. Eleven studies included fewer than 50 participants, giving rise to the possibility of small study effects. The four largest studies of diabetes (involving more than 1000 cases and controls), however, showed a clear association between enterovirus infection and clinical diabetes.

Strengths and weaknesses in relation to other studies A previous meta-analysis of coxsackie B virus serological studies found no significant association between type 1 diabetes and serology positivity, ${ }^{26}$ though summary estimates were not calculated because of significant heterogeneity between studies. Several major differences between the two meta-analyses could explain the discrepant findings. Firstly, most studies included in our review detected most enteroviruses by using PCR primers targeting the highly conserved 5 untranslated region of the enterovirus genome, whereas serological studies examined only certain serotypes. Secondly, molecular methods for detection of enteroviruses are significantly more sensitive than serology. ${ }^{555}$ Thirdly, the detection of enterovirus RNA or vp1 identifies only current or recent infection. The latter is also a limitation of molecular methods, though this would probably cause bias towards under-reporting of infection rates and estimation of a lower than actual effect size. We could not examine whether participants had multiple enterovirus infections or the same persistent infection before the development of autoimmunity or type 1 diabetes.

Autoimmunity was mostly defined as a positive result for at least one autoantibody associated with 
Table $\mathbf{5}$ | Summary odds ratios of diabetes studies including sensitivity analyses

\begin{tabular}{lcccc} 
& No of studies & OR $(95 \% \mathrm{Cl})$ & $\mathrm{P}$ value & $\mathrm{X}^{2} / \mathrm{df}^{*}$ \\
Diabetes (all studies) & 25 & $9.8(5.5$ to 17.4$)$ & $<0.001$ & 3.21 \\
\hline Method of virus detection: & & & & \\
\hline RNA & 21 & $8.8(4.7$ to 16.7$)$ & $<0.001$ & 3.37 \\
\hline vp1 & 4 & $15.4(7.5$ to 31.5) & $<0.001$ & 0.35 \\
\hline Case definition: & & & & \\
\hline Newly diagnosed & 12 & $12.7(6.4$ to 25.2$)$ & $<0.001$ & 2.44 \\
\hline Established (including recent onset) & 11 & $10.8(4.0$ to 29.4$)$ & $<0.001$ & 2.62 \\
\hline Eventual & 2 & $1.3(0.2$ to 6.9$)$ & 0.79 & 1.60 \\
\hline Study location: & & & & \\
\hline Europe only & 19 & $8.6(4.3$ to 17.3$)$ & $<0.001$ & 3.75 \\
\hline Non-European countries & 6 & $13.5(7.1$ to 25.8$)$ & $<0.001$ & 0.34 \\
\hline Study quality: & & & & \\
\hline$\quad$ NOS score $\geq 5$ & 18 & $9.0(4.6$ to 17.5$)$ & $<0.001$ & 3.77 \\
\hline
\end{tabular}

NOS=Newcastle-Ottawa

${ }^{\star}$ Cochrane $X^{2}$ divided by degrees of freedom. Values $>1$ indicate heterogeneity.

type 1 diabetes, and the presence of a single antibody does not confer a high lifetime risk of clinical diabetes compared with positive results for multiple anti bodies. ${ }^{567}$ Prospective studies are also limited by the frequency of sample collection, which might be only six or 12 months apart, and it is noteworthy that the only prospective study reporting an odds ratio under 1 had the longest sampling intervals. ${ }^{22}$ A temporal association between seroconversion to autoimmunity and infection could be under-reported because of lack of sampling at the time of infection or seroconversion, or both, in some individuals.

Maternal enterovirus infection might also be a risk factor for autoimmunity and type 1 diabetes. We did not specify maternal infection in our inclusion criteria, though among the "eventual diabetes" group enterovirus RNA was more commonly detected in dried blood spots from newborn infants who subsequently developed type 1 diabetes. Two of the included studies in the pre-diabetes group examined maternal enterovirus infection by using serology and showed little or no association between infection and subsequent development of autoimmunity in their offspring. ${ }^{2028}$

There is conflicting evidence as to whether the presence or absence of high risk HLA genotypes modifies the association between enterovirus infection and type 1 diabetes. Several groups have reported higher rates of enterovirus infection in children with low risk HLA genotypes. ${ }^{1658}$ Unfortunately, we could not do a subgroup analysis by HLA genotype in the diabetes

\section{WHAT IS ALREADY KNOWN ON THIS TOPIC}

Observational studies have shown an association between enteroviruses and type 1 diabetes

A systematic review of serological studies only found no association

\section{WHAT THIS STUDY ADDS}

A review of molecular studies showed an association between current enterovirus infection and type 1 diabetes studies because most studies did not do HLA genotyping in control participants. In the pre-diabetes group, the odds ratio of enterovirus infection in high risk HLA participants (3.5) was not different from the overall odds ratio (3.4), and the conflicting results from the two studies with low risk participants do not support an association between enterovirus infection and autoimmunity. Ideally, future studies should include individuals with low risk HLA genotypes to explore whether genetic risk modifies the effect of enterovirus infection on the risk of type 1 diabetes.

\section{Conclusion}

Our results show an association between type 1 diabetes and enterovirus infection, with a more than nine times the risk of infection in cases of diabetes and three times the risk in children with autoimmunity. The odds of having an enterovirus infection in people with established diabetes (odds ratio 11) suggest that persistent enterovirus infection is also common among patients with type 1 diabetes. While it is not possible to determine a causal relation between infection and type 1 diabetes with a randomised controlled trial, larger multicentre international prospective studies could examine interactions between type 1 diabetes and various environmental, geographical, and genetic factors.

Contributors: WGY performed the literature search, extracted, analysed, and interpreted the data, and drafted the article. MEC conceived and designed the review, helped extract and interpret the data, and revised the article critically for intellectual content. WDR revised the article critically for intellectual content and approved the final draft for publication. WDR is guarantor.

Funding: This research received no specific grant from any funding agency in the public, commercial, or not-for-profit sectors.

Competing interests: All authors have completed the Unified Competing Interest form at www.icmje.org/coi_disclosure.pdf (available on request from the corresponding author) and declare: no support from any organisation for the submitted work; no financial relationships with any organisations that might have an interest in the submitted work in the previous 3 years; no other relationships or activities that could appear to have influenced the submitted work.

Ethical approval: Not required

Data sharing: No additional data available.

1 Hober D, Sauter P. Pathogenesis of type 1 diabetes mellitus: interplay between enterovirus and host. Nat Rev Endocrinol 2010;6:279-89.

2 Diamond PG. Incidence and trends of childhood type 1 diabetes worldwide 1990-1999. Diabet Med 2006;23:857-66.

3 EURODIAB. Variation and trends in incidence of childhood diabetes in Europe. Lancet 2000;355:873-6.

4 Patterson C, Dahlquist G, Gyürüs E, Green A, Soltész G. Incidence trends for childhood type 1 diabetes in Europe during 1989-2003 and predicted new cases 2005-20: a multicentre prospective registration study. Lancet 2009;373:2027-33.

5 Chong J, Craig M, Cameron F, Clarke C, Rodda C, Donath S, et al. Marked increase in type 1 diabetes mellitus incidence in children aged 0-14 yrs in Victoria, Australia, from 1999 to 2002. Pediatr Diabetes 2007;8:67-73.

6 Taplin C, Craig M, Lloyd M, Taylor C, Crock P, Silink M, et al. The rising incidence of childhood type 1 diabetes in New South Wales, 19902002. Med J Aust 2005;183:243.

7 Green A, Patterson C. Trends in the incidence of childhood-onset diabetes in Europe 1989-1998. Diabetologia 2001;44:3-8.

8 Kimpimaki T, Kupila A, Hamalainen AM, Kukko M, Kulmala P, Savola K, et al. The first signs of B-cell autoimmunity appear in infancy in genetically susceptible children from the general population: the Finnish Type 1 Diabetes Prediction and Prevention Study. J Clin Endocrinol Metab 2001;86:4782-8. 
9 Wagenknecht LE, Roseman JM, Herman WH. Increased incidence of insulin-dependent diabetes mellitus following an epidemic of coxsackievirus B5. Am I Epidemiol 1991;133:1024-31.

10 Gamble D, Kinsley M, Fitzgerald M, Bolton R, Taylor K. Viral antibodies in diabetes mellitus. BMJ 1969;3:627.

11 Helfand RF, Gary HE Jr, Freeman CY, Anderson LJ, Pallansch MA. Serologic evidence of an association between enteroviruses and the onset of type 1 diabetes mellitus. Pittsburgh Diabetes Research Group. J Infect Dis 1995;172:1206-11.

12 Nairn C, Galbraith DN, Taylor KW, Clements GB. Enterovirus variants in the serum of children at the onset of type 1 diabetes mellitus. Diabet Med 1999;16:509-13.

13 Lönnrot M, Korpela K, Knip M, Ilonen J, Simell O, Korhonen S, et al. Enterovirus infection as a risk factor for beta-cell autoimmunity in a prospectively observed birth cohort: the Finnish Diabetes Prediction and Prevention Study. Diabetes 2000;49:1314.

14 Andreoletti L, Hober D, Hober-Vandenberghe C, Belaich S, Vantyghem MC, Lefebvre J, et al. Detection of coxsackie B virus RNA sequences in whole blood samples from adult patients at the onset of type I diabetes mellitus. J Med Virol 1997;52:121-7.

15 Craig ME, Howard NJ, Silink M, Rawlinson WD. Reduced frequency o HLA DRB $1^{*} 03-D Q B 1 * 02$ in children with type 1 diabetes associated with enterovirus RNA. J Infect Dis 2003;187:1562-70.

16 Sarmiento L, Cabrera-Rode E, Lekuleni L, Cuba I, Molina G, Fonseca $M$, et al. Occurrence of enterovirus RNA in serum of children with newly diagnosed type 1 diabetes and islet cell autoantibodypositive subjects in a population with a low incidence of type 1 diabetes. Autoimmunity 2007;40:540-5.

17 Richardson S, Willcox A, Bone A, Foulis A, Morgan N. The prevalence of enteroviral capsid protein vp1 immunostaining in pancreatic islets in human type 1 diabetes. Diabetologia 2009;52:1143-51.

18 Hyöty H, Hiltunen M, Knip M, Laakkonen M, Vähäsalo P, Karjalainen J, et al. A prospective study of the role of coxsackie B and other enterovirus infections in the pathogenesis of IDDM. Childhood Diabetes in Finland (DiMe) Study Group. Diabetes 1995;44:652.

19 Hiltunen M, Hyöty H, Knip M, Ilonen J, Reijonen H, Vähäsalo P, et al. Islet cell antibody seroconversion in children is temporally associated with enterovirus infections. J Infect Dis 1997;175:554-60.

20 Salminen K, Sadeharju K, Lönnrot M, Vähäsalo P, Kupila A Korhonen S, et al. Enterovirus infections are associated with the induction of beta-cell autoimmunity in a prospective birth cohort study. J Med Virol 2003;69:91-8.

21 Tuvemo T, Dahlquist G, Frisk G, Blom L, Friman G, Landin-Olsson M, et al. The Swedish childhood diabetes study III: IgM against coxsackie $B$ viruses in newly diagnosed type 1 (insulin-dependent) diabetic children-no evidence of increased antibody frequency. Diabetologia 1989;32:745-7.

22 Graves P, Rotbart H, Nix W, Pallansch M, Erlich H, Norris J, et al. Prospective study of enteroviral infections and development of betacell autoimmunity: diabetes autoimmunity study in the young (DAISY). Diabetes Res Clin Pract 2003;59:51-61.

23 Hadden D, Connolly J, Montgomery D, Weaver J. Coxsackie B virus diabetes. BMJ 1972;4:729.

24 Juhela S, Hyoty H, Roivainen M, Harkonen T, Putto-Laurila A, Simell O, et al. T-cell responses to enterovirus antigens in children with type 1 diabetes. Diabetes 2000;49:1308-13.

25 Filippi CM, von Herrath MG. Viral trigger for type 1 diabetes: pros and cons. Diabetes 2008; $57: 2863-71$

26 Green J, Casabonne D, Newton R. Coxsackie B virus serology and type 1 diabetes mellitus: a systematic review of published case-control studies. Diabet Med 2004;21:507-14.

27 Wells G, Shea BO, Connell D. The Newcastle-Ottawa Scale (NOS) for assessing the quality of nonrandomized studies in meta-analysis. 2010. www.ohri.ca/programs/clinical_epidemiology/oxford.asp.

28 Sadeharju K, Hamalainen AM, Knip M, Lonnrot M, Koskela P, Virtanen SM, et al. Enterovirus infections as a risk factor for type I diabetes: virus analyses in a dietary intervention trial. Clin Exp Immunol 2003;132:271-7.

29 Salminen KK, Vuorinen T, Oikarinen S, Helminen M, Simell S, Knip M, et al. Isolation of enterovirus strains from children with preclinical type 1 diabetes. Diabet Med 2004;21:156-64.

30 Al-Shaheeb A, Talati R, Catteau J, Sanderson K, Niang Z, Rawlinson W, et al. Enterovirus infections are common in children at risk of type 1 diabetes and associated with transient and persistent autoimmunity. Diabetes 2010;59(suppl 1):A 67-OR.

31 Lönnrot M, Salminen K, Knip M, Savola K, Kulmala P, Leinikki P, et al. Enterovirus RNA in serum is a risk factor for beta-cell autoimmunity and clinical type 1 diabetes: a prospective study. J Med Virol 2000;61:214-20.

32 Coutant R, Palmer P, Carel JC, Cantero-Aguilar L, Lebon P, Bougneres PF. Detection of enterovirus RNA sequences in serum samples from autoantibody-positive subjects at risk for diabetes. Diabet Med 2002:19:968-9.

33 Moya-Suri V, Schlosser M, Zimmermann K, Rjasanowski I, Gurtler L, Mentel R. Enterovirus RNA sequences in sera of schoolchildren in the general population and their association with type 1-diabetesassociated autoantibodies. J Med Microbiol 2005;54:879-83.

34 Maha MM, Ali MA, Abdel-Rehim SE, Abu-Shady EA, El-Naggar BM, Maha YZ. The role of coxsackieviruses infection in the children of insulin dependent diabetes mellitus. J Egyptian Public Health Assoc 2003;78:305-18.

35 Foy CA, Quirke P, Lewis FA, Futers TS, Bodansky HJ. Detection of common viruses using the polymerase chain reaction to assess levels of viral presence in type 1 (insulin-dependent) diabetic patients. Diabet Med 1995;12:1002-8.

36 Dahlquist G, Forsberg J, Hagenfeldt L, Boman J, Juto P. Increased prevalence of enteroviral RNA in blood spots from newborn children who later developed type 1 diabetes: a population-based casecontrol study. Diabetes Care 2004;27:285-6.

37 Dotta F, Censini S, van Halteren A, Marselli L, Masini M, Dionisi S et al. Coxsackie B4 virus infection of cells and natural killer cell insulitis in recent-onset type 1 diabetic patients. Proc Natl Acad Sci USA 2007;104:5115.

38 Foulis AK, Farquharson MA, Cameron SO, McGill M, Schönke H, Kandolf R. A search for the presence of the enteroviral capsid protein VP1 in pancreases of patients with type 1 (insulin-dependent) diabetes and pancreases and hearts of infants who died of coxsackieviral myocarditis. Diabetologia 1990;33:290-8.

39 Oikarinen M, Tauriainen S, Honkanen T, Oikarinen S, Vuori K, Kaukinen K, et al. Detection of enteroviruses in the intestine of type 1 diabetic patients. Clin Exp Immunol 2007;151:71.

40 Yin H, Berg A-K, Tuvemo T, Frisk G. Enterovirus RNA is found in peripheral blood mononuclear cells in a majority of type 1 diabetic children at onset. Diabetes 2002;51:1964-71.

41 Clements GB, Galbraith DN, Taylor KW. Coxsackie B virus infection and onset of childhood diabetes. Lancet 1995;346:221-3.

42 Ylipaasto P, Klingel K, Lindberg AM, Otonkoski T, Kandolf R, Hovi T, et al. Enterovirus infection in human pancreatic islet cells, islet tropism in vivo and receptor involvement in cultured islet beta cells. Diabetologia 2004;47:225-39.

43 Schulte BM, Bakkers I, Lanke KHW, Melchers WIG, Westerlaken C, Allebes W, et al. Detection of enterovirus RNA in peripheral blood mononuclear cells of type 1 diabetic patients beyond the stage of acute infection. Viral Immunol 2010;23:99-104.

44 Toniolo A, Maccari G, Federico G, Salvatoni A, Bianchi G, Baj A. Are enterovirus infections linked to the early stages of type 1 diabetes? American Society for Microbiology Meeting, San Diego, CA; 23-27 May 2010 (abstract)

45 Yoon JW, Austin M, Onodera T, Notkins AL. Isolation of a virus from the pancreas of a child with diabetic ketoacidosis. N Engl J Med 1979;300:1173-9.

46 Dotta F, Censini S, van Halteren A, Marselli L, Masini M, Dionisi S, et al. Coxsackie B4 virus infection of cells and natural killer cell insulitis in recent-onset type 1 diabetic patients. Proc Natl Acad Sci 2007:104:5115.

47 Jacques J, Moret H, Minette D, Leveque N, Jovenin N, Deslee G, et al. Epidemiological, molecular, and clinical features of enterovirus respiratory infections in French children between 1999 and 2005. J Clin Microbiol 2008;46:206.

48 Tseng F, Huang H, Chi C, Lin T, Liu C, Jian J, et al. Epidemiological survey of enterovirus infections occurring in Taiwan between 2000 and 2005: analysis of sentinel physician surveillance data. J Med Virol 2007;79:1850-60.

49 Luopajärvi K, Savilahti E, Virtanen S, Ilonen J, Knip M, Åkerblom H, et al. Enhanced levels of cow's milk antibodies in infancy in children who develop type 1 diabetes later in childhood. Pediatr Diabetes 2008;9:434-41.

50 Bener A, Alsaied A, Al-Ali M, Al-Kubaisi A, Basha B, Abraham A, et al. High prevalence of vitamin $D$ deficiency in type 1 diabetes mellitus and healthy children. Acta Diabetol 2009;46:183-89.

51 Couper J, Beresford S, Hirte C, Baghurst P, Pollard A, Tait B, et al. Weight gain in early life predicts risk of islet autoimmunity in children with a first-degree relative with type 1 diabetes. Diabetes Care 2009;32:94.

52 Doornum GJJ, Schutten M, Voermans J, Guldemeester GJJ, Niesters HGM. Development and implementation of real-time nucleic acid amplification for the detection of enterovirus infections in comparison to rapid culture of various clinical specimens. / Med Virol 2007;79:1868-76.

53 Rotbart HA, Hayden FG. Picornavirus infections: a primer for the practitioner. Arch Fam Med 2000;9:913-20.

54 Van Doornum G, Schutten M, Voermans J, Guldemeester G, Niesters H. Development and implementation of real-time nucleic acid amplification for the detection of enterovirus infections in comparison to rapid culture of various clinical specimens. I Med Virol 2007;79:1868-76

55 Iturriza-Gómara M, Megson B, Gray J. Molecular detection and characterization of human enteroviruses directly from clinical samples using RT-PCR and DNA sequencing. J Med Virol 2006;78:243-53. 
56 Barker JM, Barriga KJ, Yu L, Miao D, Erlich HA, Norris JM, et al. Prediction of autoantibody positivity and progression to type 1 diabetes: Diabetes Autoimmunity Study in the Young (DAISY). J Clin Endocrinol Metab 2004;89:3896-902.

57 Hummel M, Bonifacio E, Schmid S, Walter M, Knopff A, Ziegler A. Brie communication: early appearance of islet autoantibodies predicts childhood type 1 diabetes in offspring of diabetic parents. Ann Intern Med 2004;140:882.

58 Craig ME, Howard NJ, Silink M, Rawlinson WD. Reduced frequency of HLA DRB1*03-DQB1*02 in children with type 1 diabetes associated with enterovirus RNA. J Infect Dis 2003;187:1562-70.
59 NHMRC. NHMRC additional levels of evidence and grades for recommendations for developers of guidelines. Australian Government, 2009.

60 Buesa-Gomez J, de la Torre JC, Dyrberg T, Landin-Olsson M, Mauseth RS, Lernmark A, et al. Failure to detect genomic viral sequences in pancreatic tissues from two children with acute-onse diabetes mellitus. J Med Virol 1994;42:193-7.

61 Kawashima H, Ihara T, loi H, Oana S, Sato S, Kato N, et al. Enterovirus-related type 1 diabetes mellitus and antibodies to glutamic acid decarboxylase in Japan. J Infect 2004;49:147-51.

Accepted: 25 October 2010 